\title{
Contributions of short-range and excluded-volume interactions to unperturbed polymer chain dimensions
}

\author{
$\operatorname{AUTHOR}(\mathrm{S}):$ \\ Yamakawa, H; Yoshizaki, T
}

\section{CITATION:}

Yamakawa, $\mathrm{H}$... [et al]. Contributions of short-range and excluded-volume interactions to unperturbed polymer chain dimensions. JOURNAL OF CHEMICAL PHYSICS 2004, 121(7): 3295-3298

\section{ISSUE DATE:}

2004-08-15

URL:

http://hdl.handle.net/2433/50084

\section{RIGHT:}

Copyright 2004 American Institute of Physics. This article may be downloaded for personal use only. Any other use requires prior permission of the author and the American Institute of Physics. 


\title{
Contributions of short-range and excluded-volume interactions to unperturbed polymer chain dimensions
}

\author{
Hiromi Yamakawa and Takenao Yoshizaki \\ Department of Polymer Chemistry, Kyoto University, Katsura, Kyoto 615-8510, Japan
}

(Received 9 March 2004; accepted 26 May 2004)

\begin{abstract}
A Monte Carlo study is made of the mean-square radius of gyration $\left\langle S^{2}\right\rangle$ for the freely rotating chain with such fictitious excluded-volume interactions that the Lennard-Jones 6-12 potentials at the $\Theta$ temperature act only between the fourth-through $(3+\Delta)$ th-neighbor beads $(\Delta \geqslant 1)$ along the chain. The behavior of the asymptotic value $\left(\left\langle S^{2}\right\rangle / n\right)_{\infty}$ of the ratio $\left\langle S^{2}\right\rangle / n$ as a function of the number $n$ of bonds in the chain in the limit of $n \rightarrow \infty$ is examined as a function of $\Delta$. It is shown that the approach of $\left(\left\langle S^{2}\right\rangle / n\right)_{\infty}$ to its value for the real unperturbed chain with $\Delta=\infty$ is so slow that the interactions between even up to about 100th-neighbor beads should be taken into account in order to reproduce nearly its dimension. The result implies that the unperturbed polymer chain dimension as experimentally observed at the $\Theta$ temperature depends not only on short-range interactions but also to a considerable extent on the long-range excluded-volume interactions, and that the asymptotic value $C_{\infty}$ of the characteristic ratio $C_{n}$ for the rotational isomeric state model in the limit of $n \rightarrow \infty$, which is determined only by the very local conformational energy, cannot be directly compared with the corresponding experimental value. (C) 2004 American Institute of Physics.
\end{abstract}

[DOI: $10.1063 / 1.1774155]$

\section{INTRODUCTION}

A Monte Carlo (MC) study ${ }^{1}$ has recently been made of effects of chain stiffness and chain ends on the gyrationradius expansion factor $\alpha_{S}$ of a polymer chain as defined as the square root of the ratio of the mean-square radius of gyration $\left\langle S^{2}\right\rangle$ to its unperturbed value $\left\langle S^{2}\right\rangle_{0}$ on the basis of the freely rotating chain with fixed bond lengths and bond angles (short-range interactions) and with (long-range) excluded-volume interactions ${ }^{2}$ as represented by the Lennard-Jones (LJ) 6-12 potential between beads (segments) in a cutoff version. Here, the unperturbed state or the $\Theta$ temperature for the model chain has been determined so that the ratio $\left\langle S^{2}\right\rangle / n$ as a function of the number $n$ of bonds in the chain becomes a constant independent of $n$ in the limit of $n \rightarrow \infty$ (strictly for large but finite $n$ ). (Note that this $\Theta$ temperature, which corresponds to the experimental one, is different from that ${ }^{3}$ determined on the basis of the concept of the tricritical point.) It has then been found that the asymptotic value of $\left\langle S^{2}\right\rangle_{0} / n$ in this limit from the MC data at the $\Theta$ temperature is appreciably (ca. 20\%) larger than that for the ideal (freely rotating) chain without any (higher-order short-range and excluded-volume) interactions. This indicates that the unperturbed polymer chain dimension as experimentally observed may be considerably affected by nonbonded interactions, both short-range and excluded-volume, as already pointed out by Bruns ${ }^{4}$ on the basis of his MC results for a self-avoiding walk on a simple cubic lattice with nearest-neighbor attractive cites.

If the above asymptotic behavior is governed by shortrange and excluded-volume interactions between beads which are not very far from each other along the chain, the latter interactions at the $\Theta$ temperature may be regarded as of "short range" without contradiction to the prevailing notion that the unperturbed $(\Theta)$ state of a polymer chain may be determined only by short-range interactions. Otherwise, such a notion should be altered.

Now, in the rotational isomeric state (RIS) model, ${ }^{5}$ (higher-order) short-range interactions consist of those between unbonded atoms and groups associated with its two successive coupled RISs, and all conformational properties may be determined only by their statistical weights (along with bond lengths and bond angles). Local conformational properties may then be considered to be rather well described in terms of parameters for such short-range interactions. However, the RIS model (or the above notion) may possibly break down for a strictly quantitative explanation of global conformational properties such as the asymptotic value of $\left\langle S^{2}\right\rangle_{0} / n$ or that, $C_{\infty}$, of the characteristic ratio $C_{n}$ in the limit of $n \rightarrow \infty$. (Recall that $C_{n}$ is defined as the unperturbed mean-square end-to-end distance divided by $n l^{2}$ with $l$ the bond length..$^{5}$ ) The purpose of the present paper is to clarify whether the excluded-volume interactions at the $\Theta$ temperature may be regarded as of short range or not.

For this purpose, we carry out a MC simulation by the use of the freely rotating chain with such fictitious excludedvolume interactions that the cutoff LJ potentials at the $\Theta$ temperature act only between the fourth-through $(3+\Delta)$ th-neighbor beads $(\Delta \geqslant 1)$ along the chain. We note that the interactions between the third-neighbor beads have been neglected, since they seem to make the chain locally take the cis conformation to excess ${ }^{1}$ and therefore to make the chain dimension smaller (apparently make the chain more flexible). If MC data for $\left\langle S^{2}\right\rangle / n$ for this fictitious chain with small $\Delta$ agree very nearly with the previous MC data for $\left\langle S^{2}\right\rangle_{0} / n$ for the real chain with the full potential, the excluded-volume interactions at the $\Theta$ temperature is of 
short range. Otherwise, the unperturbed polymer chain dimension cannot be determined only by the short-range interactions, indicating that it has little physical significance to determine the statistical weights of the coupled RISs from the detailed conformational energy map so as to obtain the agreement between the experimental and RIS theoretical values of $C_{\infty}$.

\section{MODEL AND METHODS}

The model chain used in this MC study is the same as that used in the previous paper ${ }^{1}$ on $\alpha_{S}$ except for its total potential energy. It is the freely rotating chain ${ }^{2,5}$ composed of $n$ bonds, each of length unity, and of $n+1$ identical beads, whose centers are located at the $n-1$ junctions of two successive bonds and at the two terminal ends. The beads are numbered $0,1,2, \ldots, n$ from one end to the other, and the $i$ th bond vector connects the centers of the $(i-1)$ th and $i$ th beads with its direction from the $(i-1)$ th to the $i$ th bead. All the $n-1$ bond angles $\theta$ (not supplements) are fixed at $\theta$ $=109^{\circ}$, so that the configuration of the entire chain may be specified by the set of $n-2$ internal rotation angles $\left\{\phi_{n-2}\right\}$ $=\left(\phi_{2}, \phi_{3}, \ldots, \phi_{n-1}\right)$ apart from its position and orientation in an external Cartesian coordinate system, where $\phi_{i}$ is the internal rotation angle around the $i$ th bond vector.

The total (excluded-volume) potential energy $U_{\Delta}$ of the fictitious chain with the interactions only between the fourththrough $(3+\Delta)$ th-neighbor beads $(\Delta \geqslant 1)$ as a function of $\left\{\phi_{n-2}\right\}$ may be given by

$$
U_{\Delta}\left(\left\{\phi_{n-2}\right\}\right)=\sum_{i=0}^{n-4} \sum_{j=i+4}^{\min (n, i+3+\Delta)} u\left(R_{i j}\right)
$$

with $R_{i j}$ the distance between the centers of the $i$ th and $j$ th beads. The pair potential $u(R)$ is the cutoff version of the $\mathrm{LJ}$ 6-12 potential given by

$$
\begin{aligned}
u(R) & =\infty \quad \text { for } 0 \leqslant R<c \sigma \\
& =u^{\mathrm{LJ}}(R) \quad \text { for } c \sigma \leqslant R<3 \sigma \\
& =0 \quad \text { for } 3 \sigma \leqslant R,
\end{aligned}
$$

where $u^{\mathrm{LJ}}(R)$ is the $\mathrm{LJ}$ potential given by

$$
u^{\mathrm{LJ}}(R)=4 \epsilon\left[\left(\frac{\sigma}{R}\right)^{12}-\left(\frac{\sigma}{R}\right)^{6}\right]
$$

with $\sigma$ and $\epsilon$ the collision diameter and the depth of the potential well at the minimum of $u^{\mathrm{LJ}}(R)$, respectively. We note that $u(R)$ given by Eqs. (2) is the LJ potential cutoff at the upper bound $3 \sigma$. The lower bound $c \sigma$ in Eqs. (2) has been introduced for numerical convenience; the factor $c$ is properly chosen so that the Boltzmann factor $e^{-u^{\mathrm{LJ} / k_{B} T}}$ may be regarded as numerically vanishing compared to unity, where $k_{B}$ is the Boltzmann constant and $T$ is the absolute temperature. In practice, in double-precision numerical computation, we set

$$
c=\left[2 /\left(1+\sqrt{1+36 T^{*}}\right)\right]^{1 / 6}
$$

so that $e^{-u^{\mathrm{LJ} / k_{B} T}} \leqslant 2 \times 10^{-16}$ for $0 \leqslant R<c \sigma$, where $T^{*}$ is the reduced temperature defined by $T^{*}=k_{B} T / \epsilon$. The total poten- tial energy $U_{\Delta}$ given by Eq. (1) with $n \leqslant 3+\Delta$ is identical to the $U$ for the real chain (with indistinguishable ends) considered in the previous paper, ${ }^{1}$ so that $U_{\infty}=U$.

As done in the previous study, ${ }^{1}$ a set of $10^{5}$ sample configurations $\left\{\phi_{n-2}\right\}$ of the chain has been generated in every MC run by an application of the pivot algorithm ${ }^{6,7}$ and the Metropolis method of importance sampling ${ }^{8}$ with the use of an initial configuration generated by trial and error so that all the distances between the centers of the fourth-through (3 $+\Delta$ ) th-neighbor beads are greater than or equal to $c \sigma$. In the generation of each set, we have sampled one configuration at every $M_{\text {nom }}$ (nominal) pivot steps, and $10^{5}$ configurations in total. The number $M_{\text {nom }}$ has been chosen to be ca. $2 n$. Note that $10^{5} \times M_{\text {nom }}$ pivot steps are required to carry out a $\mathrm{MC}$ run.

The mean-square radius of gyration $\left\langle S^{2}\right\rangle$ has been evaluated from

$$
\left\langle S^{2}\right\rangle=10^{-5} \sum_{\left\{\phi_{n-2}\right\}} S^{2}\left(\left\{\phi_{n-2}\right\}\right)
$$

where the sum has been taken over the $10^{5}$ sample configurations, and the squared radius of gyration $S^{2}$ as a function of $\left\{\phi_{n-2}\right\}$ for each sample configuration has been calculated from

$$
S^{2}=\frac{1}{n+1} \sum_{i=0}^{n}\left|\mathbf{r}_{i}-\mathbf{r}_{\mathrm{c} . \mathrm{m} .}\right|^{2}
$$

Here, $\mathbf{r}_{i}$ is the vector position of the center of the $i$ th bead, and $\mathbf{r}_{\mathrm{c} . \mathrm{m}}$. is that of the center of mass of the sample chain and is given by

$$
\mathbf{r}_{\mathrm{c} . \mathrm{m} .}=\frac{1}{n+1} \sum_{i=0}^{n} \mathbf{r}_{i}
$$

All MC runs have been carried out at the $\Theta$ temperature $T^{*}=3.72$ previously ${ }^{1}$ determined. For a generation of pseudorandom numbers, we have used the subroutine package MT19937 (based on the Mersenne Twister algorithm) supplied by Matsumoto and Nishimura. ${ }^{9}$

\section{RESULTS AND DISCUSSION}

Figure 1 shows double-logarithmic plots of $\left\langle S^{2}\right\rangle / n$ against $n$ at $T^{*}=3.72(\Theta)$. The open circles represent the present MC values for the indicated values of $\Delta$, and the closed ones represent the previous values ${ }^{1}$ for the full potential $(\Delta=\infty)$. The present values plotted are those of the mean of 10 independent MC results for $n=6,10,20,50$, 100 , and 200 and that of five independent MC results for $n$ $=500$ and 1000 . The value of the standard deviation for each value does not exceed $0.3 \%$. The solid curve connects smoothly the data points for each $\Delta$. The dotted line segments connect the theoretical values for the ideal freely rotating chain calculated from 


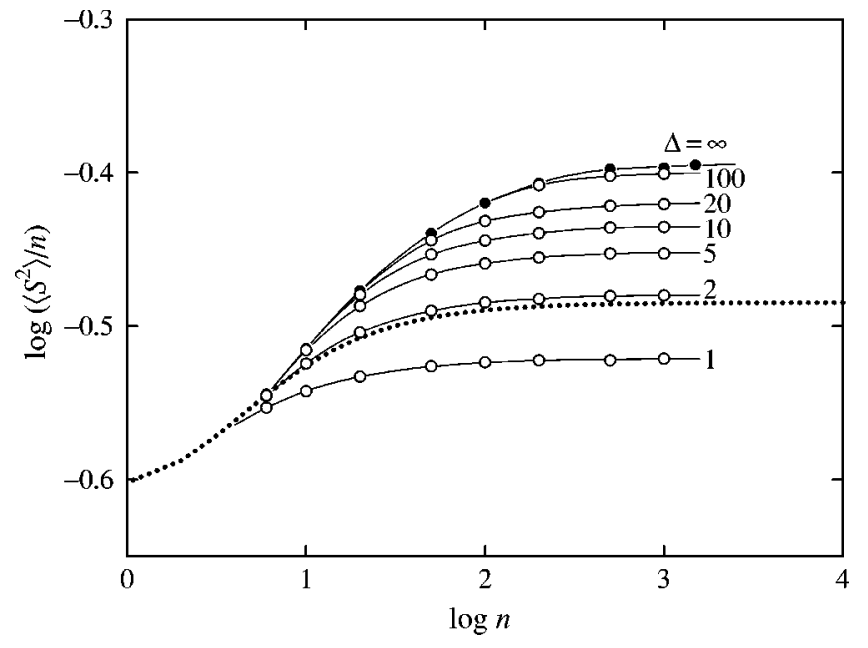

FIG. 1. Double-logarithmic plots of $\left\langle S^{2}\right\rangle / n$ against $n$ at $T^{*}=3.72(\Theta)$. The open circles represent the MC values for the indicated values of $\Delta$, and the closed ones those for $\Delta=\infty$, the solid curve connecting smoothly the data points for each $\Delta$. The dotted line segments connect the theoretical values for the ideal freely rotating chain calculated from Eq. (8) with $\theta=109^{\circ}$.

$$
\begin{aligned}
\left\langle S^{2}\right\rangle= & \frac{1}{6} \frac{1-\cos \theta}{1+\cos \theta} n+\frac{1}{6} \frac{1+6 \cos \theta-\cos ^{2} \theta}{(1+\cos \theta)^{2}} \\
& +\frac{1}{6} \frac{-1-7 \cos \theta+7 \cos ^{2} \theta+\cos ^{3} \theta}{(1+\cos \theta)^{3}} \frac{1}{n+1} \\
& -\frac{2 \cos ^{2} \theta}{(1+\cos \theta)^{4}} \frac{1-(-\cos \theta)^{n+1}}{(n+1)^{2}}
\end{aligned}
$$

with $\theta=109^{\circ}$.

For each $\Delta,\left\langle S^{2}\right\rangle / n$ increases monotonically with increasing $n$ and approaches its asymptotic value, which we denote by $\left(\left\langle S^{2}\right\rangle / n\right)_{\infty}$, as in the cases of $\Delta=\infty$ and also the ideal chain (dotted line). The values of $\left\langle S^{2}\right\rangle / n$ for $\Delta=1$, for which only the interactions between the fourth-neighbor beads are taken into account, are seen to be smaller than those for the ideal chain. This may be regarded as arising from the fact that in this case the attractive tail of the LJ potential rather than the repulsive core has a dominant effect on the chain dimension to make it smaller than that of the ideal chain. Such a tendency for $\Delta=1$ seems to be exhibited by any freely rotating chain having the interaction potential with an attractive tail between beads if the diameter of its repulsive core is not very large compared to the bond length. Although the asymptotic value $\left(\left\langle S^{2}\right\rangle / n\right)_{\infty}$ (or $\left.C_{\infty}\right)$ as a function of $\Delta$ increases monotonically with increasing $\Delta$ and approaches the value of $\left(\left\langle S^{2}\right\rangle / n\right)_{\infty}$ for $\Delta=\infty$, i.e., the value of $\left(\left\langle S^{2}\right\rangle_{0} / n\right)_{\infty}$ for the real chain, the approach of $\left(\left\langle S^{2}\right\rangle / n\right)_{\infty}$ to it is seen to be unexpectedly slow. The interactions between even up to about 100th-neighbor beads should be taken into account in order to reproduce nearly the real unperturbed chain dimension. Thus it is concluded that the excludedvolume interactions at the $\Theta$ temperature are of long range rather than of short range. In other words, the unperturbed polymer chain dimension as experimentally observed at the $\Theta$ temperature depends not only on the short-range interac- tions but also to a considerable extent on the long-range excluded-volume interactions.

Considering the above situation, we must claim that the value of $C_{\infty}$ for the RIS model, which is determined only by the very local conformational energy, cannot be directly compared with the corresponding experimental value. However, an ad hoc adjustment of the statistical weights has been made in this model in order to obtain the agreement between the theoretical and experimental values of $C_{\infty}$. Such an easy and expedient way has no longer physical significance.

Nevertheless, as mentioned in the Introduction, the RIS model may be considered to provide still useful information about local chain conformations and therefore also conformational properties of short chains or oligomers. A good example is the prediction for the behavior of $C_{n}$ (or $\left.\left\langle S^{2}\right\rangle_{0} / n\right)$ as a function of $n$ in the range of small $n$ that in some cases it first increases with increasing $n$, then passes through a maximum, and finally decreases to its asymptotic value, while in others it increases monotonically with increasing $n$. These are consistent with experimental findings. ${ }^{10}$

Finally, it is pertinent to make a remark on an analysis of experimental data for the unperturbed polymer chain dimension $\left\langle S^{2}\right\rangle_{0}$ as a function of the degree of polymerization $x$ on the basis of the helical wormlike (HW) chain model. ${ }^{10}$ In this framework, we usually determine, from a comparison of theory with experiment with respect to $\left\langle S^{2}\right\rangle_{0} / x$, the HW model parameters: the stiffness parameter $\lambda^{-1}$ representing the stiffness of the chain as an elastic wire in a thermal bath, the constant differential-geometrical curvature $\kappa_{0}$ and torsion $\tau_{0}$ of its characteristic helix taken at the minimum zero of its elastic energy, and the shift factor $M_{L}$ as defined as the molecular weight per unit contour length. The (unperturbed) HW chain with values of these parameters so determined may be considered to mimic the behavior of any real polymer chain of whatever length even at the $\Theta$ temperature, since it is clear that effects of both short-range and longrange interactions as considered above are already reflected in the determined values of $\lambda^{-1}, \kappa_{0}$, and $\tau_{0}$. Thus the present problem does not concern the analysis based on the HW chain model.

\section{CONCLUSION}

Possible contributions of long-range excluded-volume interactions to the unperturbed polymer chain dimension $\left\langle S^{2}\right\rangle$ have been examined by the MC simulation of the freely rotating chain with such fictitious interactions that the LJ 6-12 potentials at the $\Theta$ temperature act only between the fourth-through $(3+\Delta)$ th-neighbor beads $(\Delta \geqslant 1)$ along the chain. It has been found that the asymptotic value $\left(\left\langle S^{2}\right\rangle / n\right)_{\infty}$ of $\left\langle S^{2}\right\rangle / n$ in the limit of $n \rightarrow \infty$ as a function of $\Delta$ approaches very slowly the value of $\left(\left\langle S^{2}\right\rangle / n\right)_{\infty}$ for $\Delta=\infty$, indicating that the unperturbed polymer chain dimension as experimentally observed at the $\Theta$ temperature depends not only on the shortrange interactions but also to a considerable extent on the long-range excluded-volume interactions. The result implies that the value of $C_{\infty}$ for the RIS model, which is determined only by the very local conformational energy, cannot be directly compared with the corresponding experimental value. 
${ }^{1}$ H. Yamakawa and T. Yoshizaki, J. Chem. Phys. 118, 2911 (2003).

${ }^{2}$ H. Yamakawa, Modern Theory of Polymer Solutions (Harper \& Row, New York, 1971), its electronic edition is available on-line at the URL: http:// www.molsci.polym.kyoto-u.ac.jp/archives/redbook.pdf

${ }^{3}$ P. Grassberger, Phys. Rev. E 56, 3682 (1997).

${ }^{4}$ W. Bruns, Macromolecules 17, 2826 (1984).

${ }^{5}$ P. J. Flory, Statistical Mechanics of Chain Molecules (Interscience, New York, 1969).
${ }^{6}$ M. Lal, Mol. Phys. 17, 57 (1969).

${ }^{7}$ N. Madras and A. D. Sokal, J. Stat. Phys. 50, 109 (1988).

${ }^{8}$ N. Metropolis, A. W. Rosenbluth, M. N. Rosenbluth, A. H. Teller, and E. Teller, J. Chem. Phys. 21, 1087 (1953).

${ }^{9}$ M. Matsumoto and T. Nishimura, ACM Trans. Model. Comput. Simul. 8, 3 (1998), see also the URL: http://www.math.keio.ac.jp/matumoto/ emt.html

${ }^{10} \mathrm{H}$. Yamakawa, Helical Wormlike Chains in Polymer Solutions (Springer, Berlin, 1997). 